\title{
literature report
}

\section{Plugging the GAP between cell polarity and cell cycle}

The establishment and maintenance of cell polarity-that is, the asymmetrical partitioning of cellular materials-is fundamental to stem-cell renewal and development. The budding yeast Saccharomyces cerevisiae is a leading model organism for studying cell polarity. Yeast cell polarity is intimately connected to the organization of the actin cytoskeleton, which guides secretion towards the budding site or tip of the growing bud, resulting in polarized cell growth (Pruyne et al, 2004). Pioneering work by Lew \& Reed has established roles for cyclin-dependent kinases (CDKs) and their cyclin partners in cell polarity; at the time of bud emergence, G1 cyclins trigger polarized growth, whereas mitotic cyclins reverse this effect during mitosis. Inactivation of CDK following mitosis triggers redistribution of the actin cytoskeleton to the neck region for cytokinesis (Fig 1; Lew \& Reed, 1993). The role of G1 cyclins in cell polarity has been known for more than ten years but the lingering problem has been the identity of their targets. Three recent studies published in The EMBO Journal (Knaus et al, 2007; Sopko et al, 2007; Zheng et al, 2007) and one in Nature Cell Biology (McCusker et al, 2007) have significantly advanced our understanding of the important substrates of G1 cyclin-CDK and their interesting mechanisms of action.

\section{G1 cyclin-CDK promotes Cdc42 activation through GEF}

For cell polarization, cell division cycle (Cdc) 42 is the 'Rome to which all roads lead'. Cdc42 is a Rho-type small GTPase, the activity of which is regulated in budding yeast by a single guaninenucleotide exchange factor (GEF) Cdc24 and the GTPase-activating proteins (GAPs) Rga1 and the highly related Rga2, Bem2 and Bem3 (Park \& Bi, 2007). Cdc42 and G1 cyclin-CDK are essential for the initial polarization of the actin cytoskeleton, and cells defective in Cdc42 activation fail to form a bud. Conversely, expression of GTP-locked, activated alleles of Cdc42 can bypass the requirement of G1 cyclinCDK and can induce multiple buds, even in its absence (Gulli et al, 2000). Thus, G1 cyclin-CDK must regulate Cdc42 at some level; however, the direct substrates have been an unresolved question.

One appealing target of CDK is Cdc24. In haploid yeast cells, Cdc24 is sequestered in the nucleus in G1 by the Far1 protein. Phosphorylation of Far1 by G1 cyclin-CDK at START leads to its degradation and allows Cdc24 to escape from the nucleus $\left(\mathrm{O}^{\prime}\right.$ Shea \& Herskowitz, 2000). Mutations that reduce the interaction between Cdc24 and Far1 allow Cdc24 to escape to the cytoplasm in the absence of G1 cyclins; however, Cdc24 fails to localize to the bud cortex and Cdc42 does not polarize (Gulli et al, 2000). Therefore, there must be CDK targets other than Far1 that are essential for

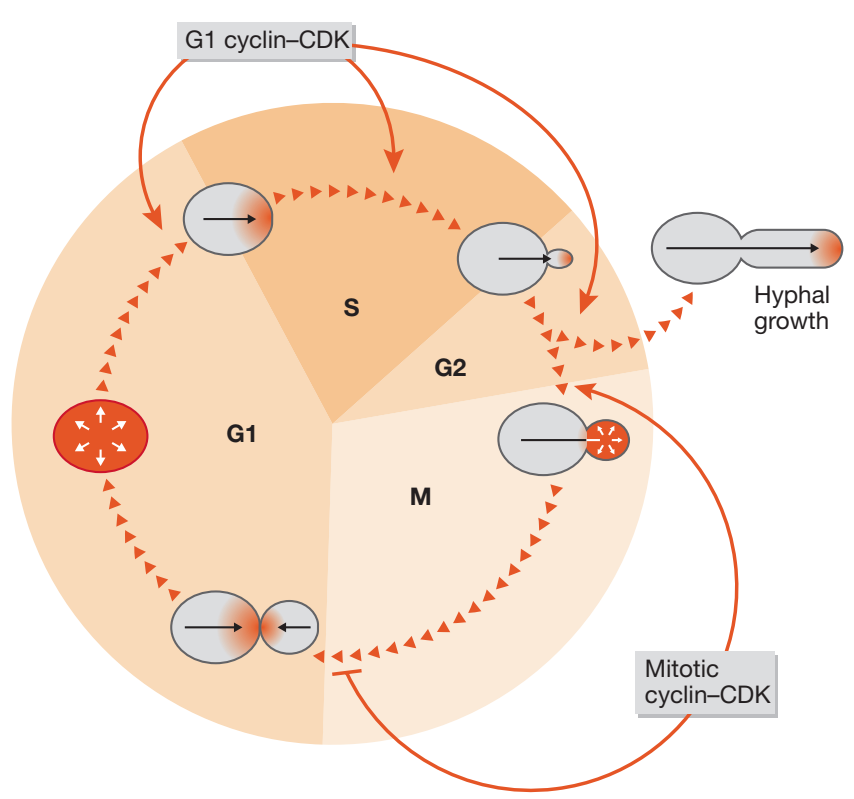

Fig 1 | Cell-cycle regulation of cell polarity in budding yeast. Polarized growth sites (red), where Cdc42 is presumably active, change during the cell cycle. These changes are brought about by the action of G1 cyclin bound to cyclin-dependent kinase (CDK) and mitotic cyclin-CDK activity. In some environments, Candida albicans keeps its polarized status and develops highly polarized hyphae.

Cdc42 polarization. Cdc24 alone is a CDK substrate in vitro; however, mutating six potential CDK-dependent phosphorylation sites of Cdc24 did not affect Cdc24 function in vivo (Moffat \& Andrews, 2004). Therefore, either Cdc24 might contain additional CDKdependent phosphorylation sites or Cdc24-associated factors are substrates of G1 cyclin-CDK.

A recent study by McCusker and colleagues using an ATPanalogue sensitive Cdc28 (CDK1) mutant has added significantly to our understanding of the role of Cdc28 in cell polarity (McCusker et al, 2007). Chemical inhibition of Cdc28 activity resulted in a rapid loss of cell polarity as judged by depolarization of the actin cytoskeleton, secretion markers and Cdc42 localization, in cells at both early and late stages of budding. This shows that Cdc28 activity is crucial for both the initiation and maintenance of cell polarity.

McCusker and colleagues also showed that, at the time of bud emergence, $\mathrm{Cdc} 24$ is in a protein complex containing the scaffolding protein Bem1 - a Cdc42 GAP Rga2 - as well as Boi1 and Boi2. Boi 1 and Boi2 are related proteins that are implicated in the regulation of Cdc42 and Rho3 in polarized exocytosis (Park \& Bi, 2007). Among the proteins in the Cdc24 complex, Boi1, Boi2 and Rga2 are good substrates of G1 cyclin-CDK both in vivo and in vitro. Importantly, a mutant form of Boi1 lacking CDK phosphorylation 


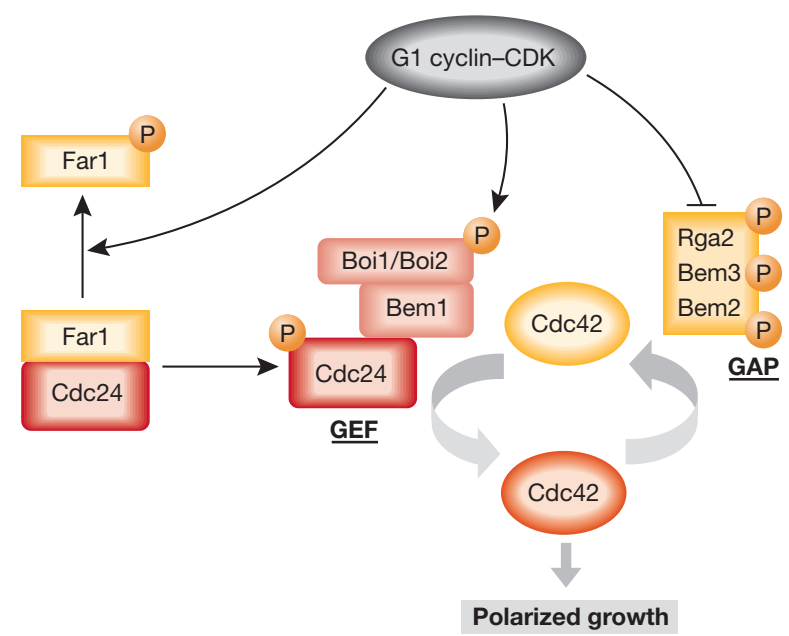

Fig 2 G1 cyclin-CDK activates Cdc42 by multiple pathways. See text for details. CDK, cyclin-dependent kinase; GAP, GTPase-activating protein; GEF, guanine-nucleotide exchange factor; $\mathrm{P}$, phosphorylation site.

sites fails to localize to the polarized site and fails to maintain polarized growth. Thus, localization of Cdc24 and its associated proteins is regulated by CDK-dependent phosphorylation. Given the essential requirement of Cdc24 activity for budding, it seems likely that Cdc2 4 activity is also increased by CDK; however, this remains to be tested.

\section{G1 cyclin-CDK inhibits Cdc42 GAPs}

Activation of Cdc24 is not the only way to activate Cdc42. Inactivation of GAPs should also lead to increased amounts of Cdc42-GTP, given that GEFs and GAPs act antagonistically on small G-proteins. However, negative regulation can be difficult to identify using genetic methods because loss-of-function effects are often more obvious than gain-of-function effects. Sopko and colleagues used a clever strategy to identify genes that are negatively regulated by the CDK Pho85 (Sopko et al, 2006). Pho85 in complex with its G1 cyclins Pcl1, Pcl2 and Pcl9 shares important overlapping roles in cell polarity with Cdc28 (Moffat \& Andrews, 2004). By using a genomewide synthetic dosage lethality screen, Sopko and colleagues found that overexpression of Rga2 kills cells lacking Pho85, suggesting that Rga2 could become hyperactive in the absence of Pho85 (Sopko et al, 2006). Sopko and colleagues have now shown that Rga2 is a true substrate of both Cdc28 and Pho85. Genetic experiments performed by these authors suggest that Rga2 catalytic activity is negatively regulated by phosphorylation through CDKs (Sopko et al, 2007).

In addition to Rga2, other Cdc42 GAPs seem to be inhibited by CDK. In a back-to-back paper published with the above study, Knaus and colleagues have shown that Cdc42 GAPs Bem2 and Bem 3 are phosphorylated at the time of bud emergence in a CDK-dependent manner (Knaus et al, 2007). Similar to Rga2, CDKdependent phosphorylation seems to inhibit Bem3 activity because expression of a BEM3-5A mutant that harbours five mutations in the consensus CDK-dependent phosphorylation sites is more toxic to the $c d c 24$ and dominant-negative FAR 1 mutants, which are partly defective in Cdc42 activation. Furthermore, Knaus and colleagues found that deletion of BEM2 triggers the formation of multiple buds in the absence of G1 cyclin-Cdc28 activity, presumably reflecting hyperactive Cdc42; therefore, Bem2 is a crucial Cdc42 GAP that must be inactivated for polarity establishment during bud-site assembly. Clearly, studies of Bem2 phosphorylation by CDK are an important subject of future research.

Inhibition of GAPs is important not only for budding but also for hyphal development in the fungal pathogen Candida albicans. Hyphae are highly polarized structures and their assembly requires sustained Cdc42 activation. A specific G1 cyclin, Hgc1, is crucial for the formation of hyphae (Court \& Sudbery, 2007; Zheng \& Wang, 2004). Zheng and colleagues have shown that the deletion of RGA2 restores the defective hyphal development in the cells that lack Hgc1, indicating that inactivation of Rga2 by Hgc1-CDK is essential for the development of hyphae (Zheng et al, 2007). Furthermore, they found that Rga2 is a substrate of Hgc1-CDK. During hyphal development, Rga2 is diffusely cytoplasmic; however, in the absence of Hgc1, Rga2 is recruited to the bud cortex under hyphae-inducing conditions. It is not clear whether Rga2 activity is also inhibited by phosphorylation, but removing a GAP from the plasma membrane sustains Cdc42 activity. Importantly, the role of Rga2 in preventing hyphal development is unique and is not shared with Bem3, suggesting that individual GAPs have specialized functions.

In summary, G1 cyclin-CDK-dependent phosphorylation promotes the activation of $\mathrm{Cdc} 42$ both by controlling - and possibly activatinga Cdc42 GEF complex and by the inhibition of Cdc42 GAPs (Fig 2).

\section{CDK is required for expansion of the bud cortex}

What is the specific role of CDK in polarized growth? Is it only important for directing growth to the emerging bud (polarization) or does it have an additional role in growth per se? A long history of experiments in yeast supports the former hypothesis. Early G1 cells-before cyclin accumulation and measurable CDK activitygrow isotropically until reaching a crucial volume. Furthermore, all previously characterized cdc28 mutants increase their cell volume under non-permissive conditions (Johnston et al, 1977), which was a criterion for defining CDC28 as a cell-cycle regulator. Finally, cells lacking Cdc28 activity are not impaired in bulk protein synthesis and do not show apparent defects in the secretory pathway (Johnston et al, 1977; McCusker et al, 2007). Thus, Cdc28 activity has been thought to be required only for polarity, and not growth.

McCusker and colleagues have now made an interesting observation that suggests a direct role for Cdc28 in cell-surface growth. They found that using a drug to inhibit Cdc28, shortly after bud emergence, not only depolarizes the cell but also slows the rate of cellsurface expansion-primarily in the bud (McCusker et al, 2007). If Cdc28 controlled only polarization, both the mother and the daughter cell should increase in surface area when Cdc28 is blocked. As expected, the growth of the bud is impaired after Cdc28 inhibition consistent with the cell polarity defect. However, the authors found that the total increase in the surface area of the mother and daughter cell was smaller after Cdc28 inhibition-after 90 minutes the authors found that it was $120 \%$ of the starting surface area, whereas it was $150 \%$ in normal cells (McCusker et al, 2007). This suggests the direct involvement of Cdc28 in cell-surface expansion.

Although this is an interesting possibility, an alternative argument can be formulated. In normal cells, cell growth occurs exclusively in the small daughter cells. However, after depolarization (Cdc28 inhibition), cell growth can occur in both mother and daughter cells. 
After depolarization, cell surface expansion in the daughter is compromised to a fractionally greater extent than the increase in mother cell. This follows simply from the different cell sizes and cell shapes: for a given volume increase, a small cell (daughter) produces more surface area than a large cell (mother) does. In addition, polarized (elongated) growth produces more surface area than isotropic (spherical) growth does. Therefore, the Cdc28 effect on polarization might explain the currently available data.

In the future, it would be interesting to determine whether CDC28 mutants have a stronger defect on the rate of cell-surface growth than other mutants that compromise cell polarity. Nevertheless, the work from McCusker and colleagues emphasizes that the maintenance of cell polarity is necessary to balance cell-surface expansion between the mother and the bud.

\section{G1 cyclin-CDK also activates Rho1}

Activation of Cdc42 is essential but not sufficient for polarized growth. Tiny buds formed by artificial activation of Cdc42, in the absence of G1 cyclins, fail to enlarge and lyse soon after bud emergence (Gulli et al, 2000). Therefore, Cdc42 is not the only target of G1 cyclin necessary for polarized growth.

Another known essential factor for bud growth is the Rho-type GTPase, Rho1. Rho1 promotes cell wall biogenesis and organizes the actin cytoskeleton during bud growth (Park \& Bi, 2007). Cells that are defective in Rho1 activation die as a result of cell lysis soon after bud emergence (Yamochi et al, 1994); therefore, Rho1 is likely to be another crucial target of G1 cyclin-CDK activity for polarized growth. Indeed, G1 cyclin-CDK seems to activate Rho1 at least in part by phosphorylating the Rho1 GEF Tus1 during bud emergence (K. Kono and Y. Ohya, personal communication). Similar to Cdc42, CDKs might regulate Rho1 at many levels; for example, the major Rho1 GAP Sac7 is a good substrate for Cdc28 (Ubersax et al, 2003)

\section{Perspectives}

The recent studies have significantly expanded our list of CDK substrates that are involved in polarized growth. A surprisingly large number of phosphorylation events on various substrates are important for cell polarity establishment and maintenance. This redundancy might have evolved to make polarization in yeast robust. These studies emphasize that Rho GTPases are crucial targets for cell-cycle regulation. Given the conservation of Cdc42 and RhoA (the Rho1 counterpart in humans) in eukaryotes, the recent findings reveal mechanisms that are likely to be broadly relevant to cell polarization in animal cells.

\section{ACKNOWLEDGEMENTS}

We thank K. Kono and Y. Ohya for sharing unpublished results, and B. Atkins, S. Buttery and K. Kono for discussions.

\section{REFERENCES}

Court H, Sudbery P (2007) Regulation of Cdc42 GTPase activity in the formation of hyphae in Candida albicans. Mol Biol Cell 18: 265-281

Gulli MP, Jaquenoud M, Shimada Y, Niederhauser G, Wiget P, Peter M (2000)

Phosphorylation of the Cdc42 exchange factor Cdc24 by the PAK-like

kinase Cla4 may regulate polarized growth in yeast. Mol Cell 6: 1155-1167
Johnston GC, Pringle JR, Hartwell LH (1977) Coordination of growth with cell division in the yeast Saccharomyces cerevisiae. Exp Cell Res 105: 79-98

Knaus M, Pelli-Gulli MP, van Drogen F, Springer S, Jaquenoud M, Peter M (2007) Phosphorylation of Bem2p and Bem3p may contribute to local activation of Cdc42p at bud emergence. EMBO / 26: 4501-4513

Lew DJ, Reed SI (1993) Morphogenesis in the yeast cell cycle: regulation by Cdc28 and cyclins. J Cell Biol 120: 1305-1320

McCusker D, Denison C, Anderson S, Egelhofer TA, Yates JR 3rd, Gygi SP, Kellogg DR (2007) Cdk1 coordinates cell-surface growth with the cell cycle. Nat Cell Biol 9: 506-515

Moffat J, Andrews B (2004) Late-G1 cyclin-CDK activity is essential for control of cell morphogenesis in budding yeast. Nat Cell Biol 6: 59-66

O'Shea EK, Herskowitz I (2000) The ins and outs of cell-polarity decisions. Nat Cell Biol 2: E39-E41

Park HO, Bi E (2007) Central roles of small GTPases in the development of cell polarity in yeast and beyond. Microbiol Mol Biol Rev 71: 48-96

Pruyne D, Legesse-Miller A, Gao L, Dong Y, Bretscher A (2004) Mechanisms of polarized growth and organelle segregation in yeast. Annu Rev Cell Dev Biol 20: $559-591$

Sopko R et al (2006) Mapping pathways and phenotypes by systematic gene overexpression. Mol Cell 21: 319-330

Sopko R, Huang D, Smith JC, Figeys D, Andrews BJ (2007) Activation of the Cdc42p GTPase by cyclin-dependent protein kinases in budding yeast. EMBO / 26: 4487-4500

Ubersax JA, Woodbury EL, Quang PN, Paraz M, Blethrow JD, Shah K, Shokat KM, Morgan DO (2003) Targets of the cyclin-dependent kinase Cdk1. Nature 425: 859-864

Yamochi W, Tanaka K, Nonaka H, Maeda A, Musha T, Takai Y (1994) Growth site localization of Rho1 small GTP-binding protein and its involvement in bud formation in Saccharomyces cerevisiae. J Cell Biol 125: 1077-1093

Zheng X, Wang Y (2004) Hgc1, a novel hypha-specific G1 cyclin-related protein regulates Candida albicans hyphal morphogenesis. EMBO / 23: 1845-1856

Zheng XD, Lee RT, Wang YM, Lin QS, Wang Y (2007) Phosphorylation of Rga2, a Cdc42 GAP, by CDK/Hgc1 is crucial for Candida albicans hyphal growth. EMBO / 26: 3760-3769

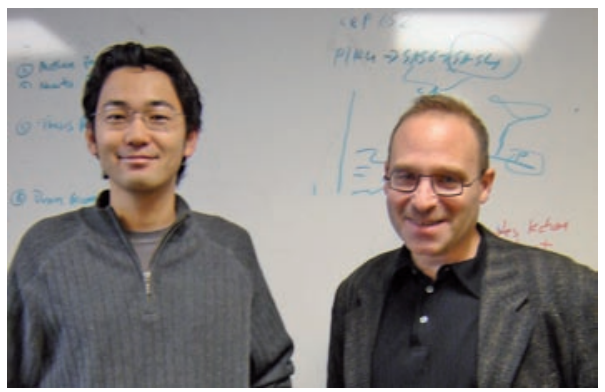

Satoshi Yoshida \& David Pellman ${ }^{+}$are at the Department of Pediatric Oncology, Dana-Farber Cancer Institute,

Harvard Medical School, 44 Binney Street, Boston,

Massachusetts 02115, USA

${ }^{+}$Corresponding author. Tel: +1617632 4918;

Fax: +1617632 6845;

E-mail:david_pellman@dfci.harvard.edu

Keywords: cell polarity; cell cycle; Cdc42; GTPase-activating protein (GAP); CDK

Submitted 15 November 2007; accepted 21 November 2007

EMBO reports (2008) 9, 39-41. doi:10.1038/sj.embor.7401142 